\title{
A Study of On-Off Keying Performance for Body Area Networks
}

\author{
Detection Techniques, Noise and Multiple Access Interference Performance
}

\author{
Igor Dotlic and Ryu Miura \\ Dependable Wireless Laboratory, Wireless Network Research Institute \\ National Institute for Information and Communications Technology \\ 3-4, Hikarino-oka, Yokosuka, 239-0847, Japan \\ \{dotlic,ryu\}@nict.go.jp
}

\begin{abstract}
In this paper we present analysis of the physical layer performance of an system with the On-Off Keying (OOK) modulation. We begin with developing a system model with describing three different receiver architectures; namely, coherent quadrature sampling receiver, envelope sampling receiver and energy detection receiver. Performances of these receiver architectures are compared in noise and Multiple Access Interference (MAI). Furthermore, the performance comparison is made in three different phases of the packet reception: preamble synchronization, Synchronization Frame Delimiter (SFD) detection and payload detection.
\end{abstract}

\section{Categories and Subject Descriptors}

C.2 [COMPUTER-COMMUNICATION NETWORKS]

General-Data communications

\section{General Terms}

Design, Performance, Interference Resiliance, Standardization

\section{Keywords}

Impulse Radio, Ultra-Wideband (UWB), Multiple Acess Interference (MAI), Interference suppression, Body Area Networks (BAN)

\section{INTRODUCTION}

Ever since in 2002 the US Federal Communications Commission (FCC) made rules for unlicensed Ultra-Wideband (UWB) band [8] Impulse-Radio UWB (IR-UWB) radio communications have been a focus of both academic research and industrial standardization efforts. In 2007 first international standards that includes IR-UWB Physical Layer (PHY) IEEE 802.15.4a-2007 [6] had been published. Recently, two

Permission to make digital or hard copies of all or part of this work for personal or classroom use is granted without fee provided that copies are not made or distributed for profit or commercial advantage and that copies bear this notice and the full citation on the first page. To copy otherwise, to republish, to post on servers or to redistribute to lists, requires prior specific permission and/or a fee.

UWBAN-2013 2013, September 30-October 02

Copyright (C) 2013 ICST 978-1-936968-89-3

DOI 10.4108/icst.bodynets.2013.253600 other 802.15 standards that include IR-UWB PHYs were published as well; namely, IEEE 802.15.4f standard for active RF-ID [9] and IEEE 802.15.6 standard for Body Area Networks (BAN) [7].

As a result of a compromise made during the standardization process IR-UWB PHY specifications in the IEEE 802.15.6 BAN standard include two different modulations; mandatory is binary Pulse Position Modulation (PPM), while Differential Phase Shift Keying (DPSK) is specified as optional. The main argument of the PPM proponents was a possibility of usage of low complexity Energy Detection (ED) receivers for the reception of the PPM modulated symbols. While ED architectures certainly exhibit low complexity and low power consumption which are very welcome qualities for any BAN system, downside is their poor performance in Multiple Access Interference (MAI). As scenarios with many co-located BANs are likely, especially in medical applications, e.g. in hospital environments, good MAI resilience is very important factor for BAN. For this reason in our previous work [5] we have introduced and analyzed differentially coherent techniques for the detection of PPM symbols which although somewhat more complex than simple ED exhibit considerably better MAI resilience.

As written above, IEEE 802.15.6 IR-UWB PHY specs [7] describe PPM as its mandatory modulation technique for the reason of possibility of ED. However, binary PPM was chosen over On-Off Keying (OOK) for ED detection not because any thorough analysis, but rather for the preferences and the interests of the parties involved in the standardization process. Nevertheless, the OOK detection exhibits roughly two times lower power consumption compared to the binary PPM detection in most receiver architectures that involve efficient duty-cycling, i.e. turning on during UWB pulse reception and off otherwise. The reason for this is that a large part of the receiver power consumption in the contemporary CMOS IC technology is in its RF part, since it operates at much higher frequency compared to the rest of the receiver. Hence, turning the receiver on at only one position per symbol in the OOK detection results in the power consumption that is approximately twice lower compared to the case of the binary PPM where the receiver is turned on twice per symbol. This is a rather large advantage of the OOK in the BAN transceivers where battery life and thus power consumption are of paramount importance.

For this reason we performed analysis of an IR-UWB system that utilizes OOK modulation with data rates that 
are similar to those used in the IEEE 802.15.6 IR-UWB PHY [7]. Besides OOK modulation, the system includes coding scheme that exhibits higher coding gain compared to the low-complexity BCH code given in the IEEE 802.15.6 IR-UWB PHY specs [7]. Synchronization Header (SHR) has similar format to the one we had proposed and which was included in the IEEE 802.15.6 specs [7] together with some modifications that we proposed in $[1,3]$. The analysis of performance is done in noise and MAI and in all three phases of the packet reception; namely, preamble synchronization, Synchronization Frame Delimiter (SFD) detection and payload detection. Three different receiver architectures were used; namely ED, fully coherent quadrature sampling receiver and Envelope Detection receiver.

\section{PRELIMINARIES}

\subsection{Transmitted Packet}

As aforesaid the packet structure is the same as in the IEEE 802.15.6 IR-UWB PHY specs [7]; SHR consists of Preamble (PR) and Synchronization Frame Delimiter (SFD). SHR is followed by Physical layer Service Data Unit (PSDU), i.e. payload. For preamble, instead of four repetitions of Kasami sequences of length 63 from [7], we used eight repetitions of Gold sequences of length 31. As we have shown before $[1,3]$ this has similar performance in the synchronization with roughly two times lower correlator complexity. Following the paradigm that we brought into IEEE 802.15.6 [7] IR-UWB PHY specs, SHR represents inversion of a single Gold sequence used in preamble.

PSDU is encoded using concatenation of the outer ReedSolomon $(63,55)$ code and inner convolutional code. Coding rate of the inner code depends on a data rate used. Between outer and inner code we used algebraic bit interleaver from [7]. Maximum free distance convolutional codes from [10] of constrained length equal to 3 are employed as inner codes.

The whole packet, i.e for $k \in\{\mathrm{PR}, \mathrm{SFD}, \mathrm{PSDU}\}$, where $k$ is the symbol index, holds the same symbol duration of $T_{\text {sym }}=1024 \mathrm{~ns}$. Linear chirp IR-UWB pulse waveform denoted $w(t)$ is used; $w(t)$ has a frequency sweep of $\Delta f_{c}=$ $520 \mathrm{MHz}$ and duration of $T_{w}=32 \mathrm{~ns}$ as specified in one of the modes of the IEEE 802.15.6 IR-UWB PHY [7]. Carrier frequency used is $f_{0}=8 \mathrm{GHz}$ Hence, waveform for the $k$-th transmitted symbol is

$$
a_{k}(t)=b_{k} \exp \left(j \varphi_{k}\right) w\left(t-k T_{s y m}\right),
$$

where $b_{k} \in\{0,1\}$ is transmitted bit and $\varphi_{k} \in\{0, \pi\}$ is pseudo-random sequence used in $k \in \mathrm{PSDU}$ to flatten the spectrum of the transmitted signal, while for $k \in\{\mathrm{PR}, \mathrm{SFD}\}$ $\varphi_{k}=0$ is set. Pseudo-random sequence used is the same Gold sequence that BAN uses in PR and SFD. Since during PSDU detection synchronization is finished, i.e. the receiver knows index of the symbol being detected and thus $\varphi_{k}$, from now on we will assume $\varphi_{k}=0$ for a whole packet in detection.

\subsection{Received Signal Model}

The symbol waveform $w(t)$ passes through a channel with the impulse response denoted $h_{c}(t)$ to produce received waveform $u(t)=w(t) \otimes h_{c}(t)$, where " $\otimes$ " denotes convolution. Hence, received signal for the $k$-th symbol denoted $r_{k}(t)$ will

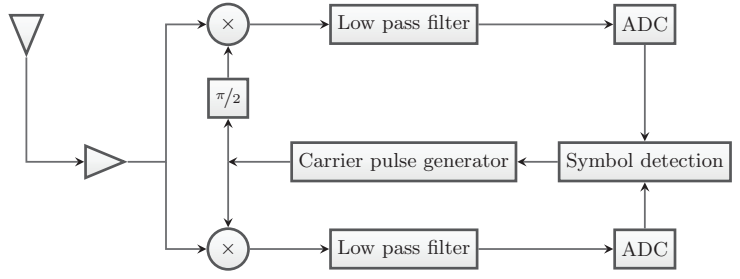

Figure 1: Quadrature sampling receiver.

have the form

$$
r_{k}(t)=b_{k} u\left(t-k T_{\text {sym }}\right) .
$$

It will be useful to define complex baseband equivalent of $r_{k}(t)$ denoted $\tilde{r}_{k}(t)$ which has a form

$$
\tilde{r}_{k}(t)=b_{k} \tilde{u}\left(t-k T_{\text {sym }}\right),
$$

where $\tilde{u}(t)$ denotes complex baseband-equivalent of $u(t)$.

\section{RECEIVER ARCHITECTURES}

\subsection{Coherent Quadrature Sampling Receiver}

Quadrature sampling receiver is shown in Fig. 1. During PSDU detection this receiver samples a window of duration $T_{I}$ with the Nyquist sampling rate. In the continuous-time representation this can be expressed as

$s_{k}(t)= \begin{cases}\tilde{r}_{k}(t), & t \in\left[\tau_{R}-T_{I} / 2+k T_{\text {sym }}, \tau_{R}+T_{I} / 2+k T_{\text {sym }}\right], \\ 0, & \text { elsewhere. }\end{cases}$

Here, $\tau_{R}$ is the timing of the receiver estimated in the synchronization process to maximize the energy of $s_{k}(t)$.

We define matched filter waveform $m(t)$ as

$$
m(t)= \begin{cases}\tilde{u}(t), & t \in\left[\tau_{R}-T_{I} / 2, \tau_{R}+T_{I} / 2\right] \\ 0, & \text { elsewhere }\end{cases}
$$

$\hat{m}(t)$ represents an estimate of $m(t)$ made during synchronization. Decision variable is formed by correlating $s_{k}(t)$ with $\hat{m}(t)$ :

$$
d_{k}=\int_{\tau_{R}-T_{I} / 2}^{\tau_{R}+T_{I} / 2} \hat{m}^{*}(t) s_{k}\left(t+k T_{s y m}\right) \mathrm{d} t .
$$

\subsection{Envelope Sampling Receiver}

Envelope sampling receiver shown in Fig. 2 samples the envelope of the received signal instead of its baseband equivalent, as quadrature sampling receiver does. The advantage of this approach is lower complexity compared to the quadrature sampling receiver described above. Hence, received signal corresponding with the $k$-th symbol is

$s_{k}^{e}(t)= \begin{cases}\left|\tilde{r}_{k}(t)\right|, & t \in\left[\tau_{R}-T_{I} / 2+k T_{\text {sym }}, \tau_{R}+T_{I} / 2+k T_{\text {sym }}\right] \\ 0, & \text { elsewhere. }\end{cases}$

Envelope matched filter waveform $m^{e}(t)$ is defined as

$$
m^{e}(t)= \begin{cases}|\tilde{u}(t)|, & t \in\left[\tau_{R}-T_{I} / 2, \tau_{R}+T_{I} / 2\right] \\ 0, & \text { elsewhere }\end{cases}
$$




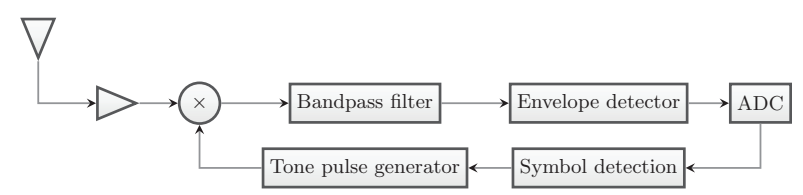

Figure 2: Envelope Sampling Receiver.

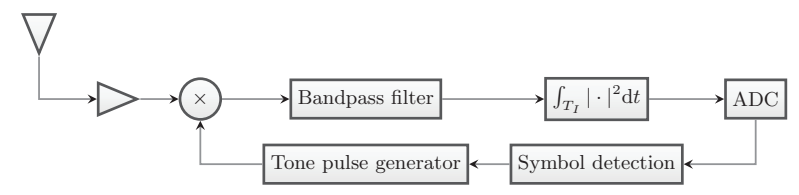

Figure 3: Energy Detection Receiver.

With $\hat{m}^{e}(t)$ we denote an estimate of $m^{e}(t)$ made during synchronization. Decision variable for $k$-th symbol is formed by correlating $s_{k}^{e}(t)$ with $\hat{m}^{e}(t)$ :

$$
d_{k}=\int_{\tau_{R}-T_{I} / 2}^{\tau_{R}+T_{I} / 2} \hat{m}(t) s_{k}^{e}\left(t+k T_{\text {sym }}\right) \mathrm{d} t .
$$

\subsection{Energy Detection Receiver}

Energy detection receiver shown in Fig. 3 represents a least complex architecture that we will study here. As Fig.3 shows, decision variable is formed by measuring the energy of the received signal in $T_{I}$

$$
d_{k}=\int_{\tau_{R}-T_{I} / 2}^{\tau_{R}+T_{I} / 2}\left|s_{k}^{e}\left(t+k T_{\text {sym }}\right)\right|^{2} \mathrm{~d} t .
$$

\subsection{Synchronization}

PE synchronization method as well as SFD detection employed are similar to the one we described in $[3,4]$ for the DPSK IR-UWB PHY of IEEE 802.15.6-2012 standard [7]. We will not give a full description here because of the lack of space. The main difference is that here no sample-wise differentiation is done as there is no information in the phase difference of the symbols. Furthermore, when quadrature sampling receiver is used synchronization is performed on complex baseband samples like in $[3,4]$. On the other side, envelope sampling receiver performs synchronization on realvalued envelope samples. For ED receiver no particular synchronization method was developed; hence, it acts as the envelope sampling receiver during the synchronization and differs from it only in the PSDU detection. After synchronization, where $\tau_{R}$ is found, each described receiver except ED calculates its corresponding matched filter estimate. For quadrature sampling receiver $\hat{m}(t)$ is calculated as follows

$$
\hat{m}(t)=\frac{1}{\left|P R \cap\left\{k \mid b_{k}=1\right\}\right|} \sum_{k \in P R} \hat{b}_{k} s_{k}\left(t+k T_{s y m}\right),
$$

for $t \in\left[\tau_{R}-T_{I} / 2+k T_{\text {sym }}, \tau_{R}+T_{I} / 2+k T_{\text {sym }}\right]$ and 0 elsewhere. Similarly, for envelope sampling receiver $\hat{m}^{e}(t)$ is calculated as

$$
\hat{m}^{e}(t)=\frac{1}{\left|P R \cap\left\{k \mid b_{k}=1\right\}\right|} \sum_{k \in P R} \hat{b}_{k} s_{k}^{e}\left(t+k T_{\text {sym }}\right),
$$

for $t \in\left[\tau_{R}-T_{I} / 2+k T_{\text {sym }}, \tau_{R}+T_{I} / 2+k T_{\text {sym }}\right]$ and 0 elsewhere.

\section{NUMERICAL RESULTS}

\subsection{General Assumptions}

Simulations are performed on the system described in Sec. 2.1. The simulations are done on IEEE 802.15.6 CM3 [11] with 100 packets transfered at every of 50 scenarios which yields 5000 packets per every simulation point. Uncoded PSDU of each packet is random between 1 and 256 octets of bits.

Simulations are done at two data rates of $218 \mathrm{Kbps}$ and $436 \mathrm{Kbps}$ which are produced by altering the coding rate of the inner convolutional code to $1 / 4$ and $1 / 2$ respectively. $T_{I}=60 \mathrm{~ns}$ is chosen for all cases studied as this value ensures that receiver exploits a large portion of multipath energy, i.e. multipath fading is low.

\subsection{Performance in Noise}

Performance in noise at $218 \mathrm{Kbps}$ and $436 \mathrm{Kbps}$ are shown in Figs. 4 and 5. From both Figs. 4 and 5 it is noticeable that coherent quadrature sampling receiver performs roughly $6 \mathrm{~dB}$ better than both envelope sampling receiver and energy detection receiver; this holds for all 3 phases of packet reception. Furthermore, from both Figs. 4 and 5 it can be seen that in payload detection there is very little difference between energy detection and envelope sampling receiver.

By comparing Figs. 4 and 5 it can be observed that coherent quadrature sampling receiver performs roughly $1 \mathrm{~dB}$ better in payload detection at the higher data rate of $436 \mathrm{Kbps}$ compared to the lower data rate of $218 \mathrm{Kbps}$. In other words, more complex coding scheme performs worst than simpler one the opposite is expected. The reason for this anomaly may be that constrained length of 3 in both inner convolutional codes used is too low for the coding rate of $1 / 4$ and higher constrained length should be employed.

For both coherent quadrature sampling receiver and envelope sampling receiver performance in synchronization is satisfying, since roughly $10 \%$ of all packets lost is due to preamble SFD misdetection.

\subsection{Performance in Multiple Access Interfer- ence}

Performance in multiple access interference is studied in environment in which both system of interest and interfering systems have packet arriving with Poisson distribution having $\lambda=150$ packs $/ \mathrm{s}$ and minimum time between packets, i.e. guard interval of $1 \mathrm{~ms}$. Examples of traffic distributions realizations for data rates of $218 \mathrm{Kbps}$ and $436 \mathrm{Kbps}$ are shown in Figs. 6 and 7 respectively. Power of all transmitters at the receiver of interest are the same, i.e. near-far ratio is $0 \mathrm{~dB}$.

Multiple access interference performance at data rates of 218 Kbps and 432 Kbps are shown in Figs. 8 and 9 respectively. It is noticeable from both Figs. that the energy detection and the envelope sampling perform considerably poorer compared to the coherent quadrature detection. However, 


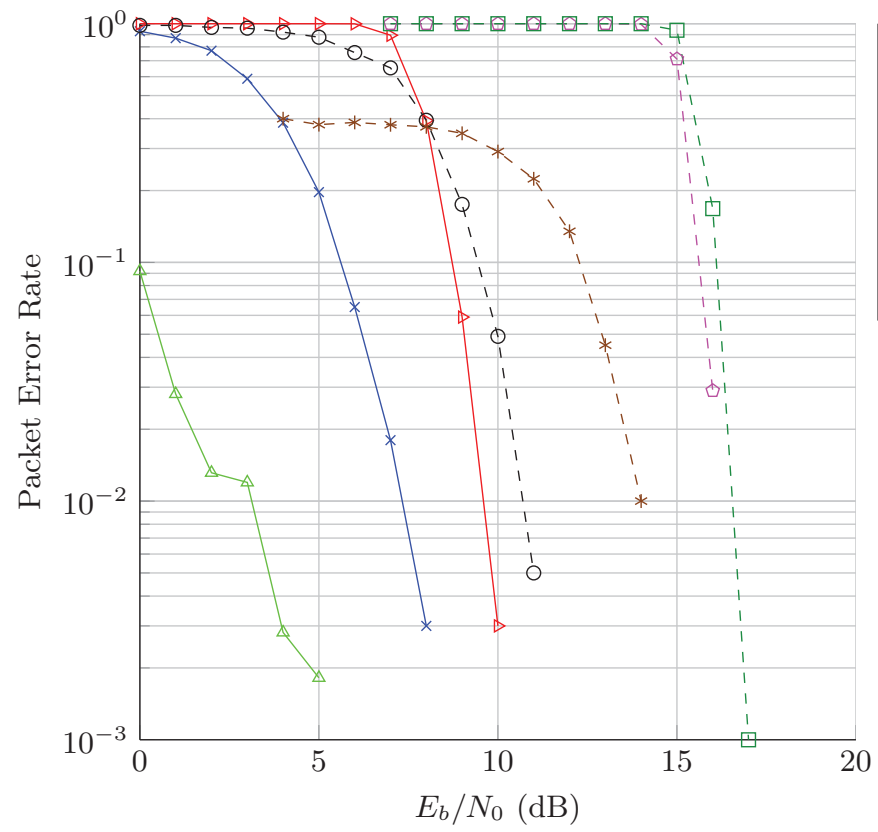

- Coherent Rec.: Preamble misdetection

$\triangle$ Coherent Rec.: SFD misdetection

$\rightarrow$ Coherent Rec.: Payload error

- $\ominus$ - Env. Det.: Preamble misdetection

- *- Env. Det.: SFD misdetection

- $\bullet-$ Env. Det.: Payload error

- - Energy. Det.: Payload error

Figure 4: Performance of receiver architectures considered in white noise at data rate of $218 \mathrm{Kbps}$

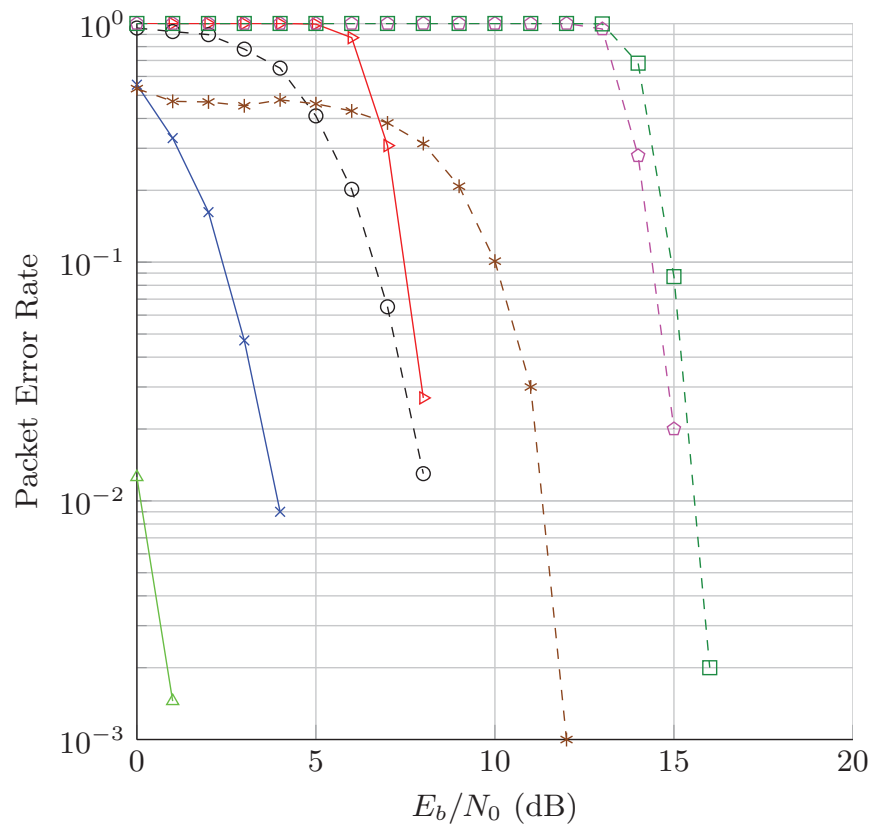

$\mp$ Coherent Rec.: Preamble misdetection

$\triangle$ Coherent Rec.: SFD misdetection

$\rightarrow$ Coherent Rec.: Payload error

- $\odot$ - Env. Det.: Preamble misdetection

-*- Env. Det.: SFD misdetection

- $\bullet-$ Env. Det.: Payload error

- $\boxminus-$ Energy. Det.: Payload error

Figure 5: Performance of receiver architectures considered in white noise at data rate of $436 \mathrm{Kbps}$. 

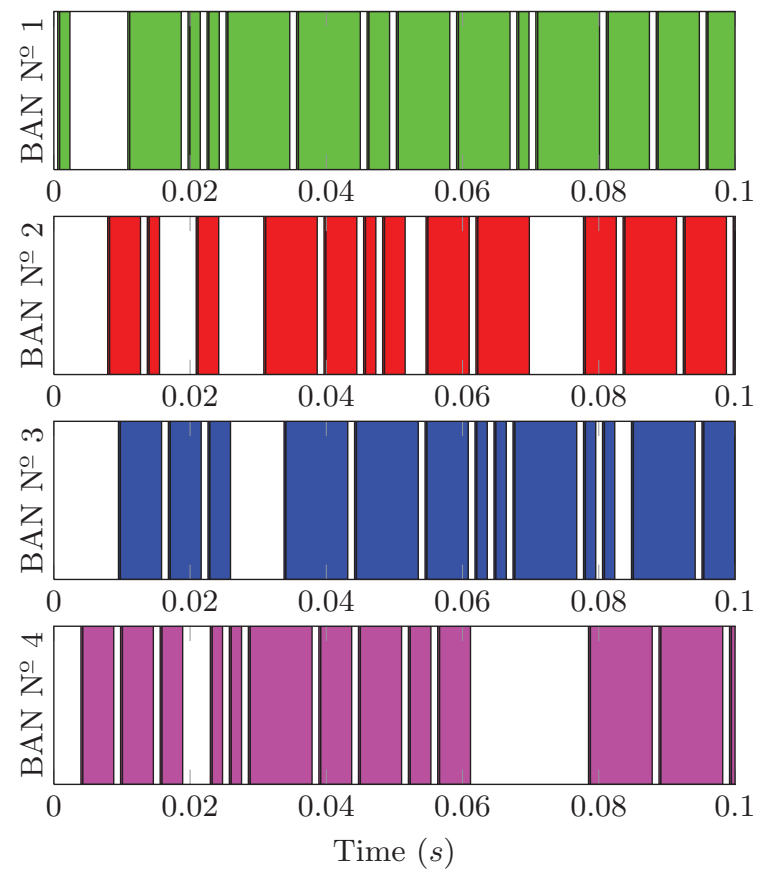

Figure 6: Multiple Access Interference traffic for data rate of $218 \mathrm{Kbps}$ and four different BANs. Shaded areas represent periods when packets are being transmitted.

in contrast with the performance in noise, in MAI there is some gain of using the envelope sampling receiver compared to the energy detection.

Furthermore, it is interesting to notice relatively poor performance of synchronization of coherent receiver compared to its performance in payload detection. The reason for this may be the fact that there is no time-hopping in the system.

\section{CONCLUSIONS}

We presented a study on performance of different receiver architectures for the OOK modulation in noise and Multiple Access Interference. From what we have shown, it can be concluded that performance in noise of the non-coherent envelope sampling and energy detection is roughly $6 \mathrm{~dB}$ poorer compared to the coherent detection. This can be regarded as acceptable loss, especially considering short ranges on which BAN devices communicate. On the other hand, in MAI noncoherent detection methods perform too poor for practical BAN applications. In other words, considering that several BANs are often co-located and that packet error rate for relatively low number of interferes becomes very high these non-coherent detection techniques are rendered impractical. In contrast, receivers with coherent detection are able to withstand considerable MAI interference. For this reason, we have to recommend coherent detection for OOK modulation in BAN systems among receiver architectures analyzed here. As a topic of future research we may mention design and analysis of differentially coherent detection methods for OOK modulation. Previously we have proposed differentially coherent architectures for DPSK and PPM modula-
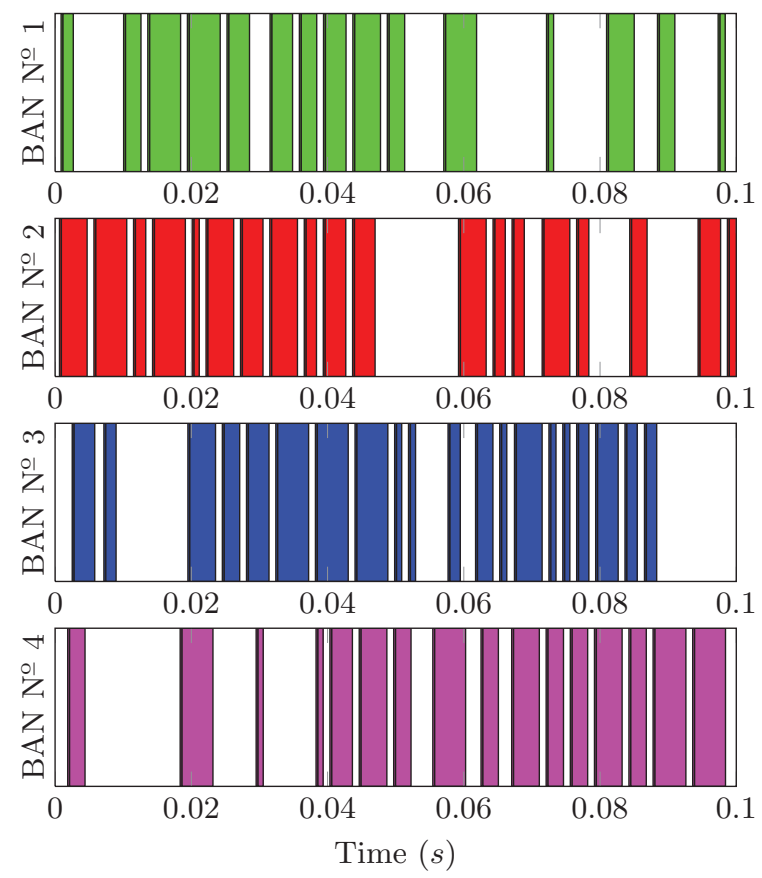

Figure 7: Multiple Access Interference traffic for data rate of $432 \mathrm{Kbps}$ and four different BANs. Shaded areas represent periods when packets are being transmitted.

tion specs in IEEE 802.15.6 IR-UWB PHY ${ }^{1}$.

\section{REFERENCES}

[1] I. Dotlić. Interference performance of IEEE 802.15.6 Impulse-Radio Ultra-Wideband physical layer. In IEEE 22nd International Symposium on Personal Indoor and Mobile Radio Communications (PIMRC), pages $2148-2152$, Sept. 2011.

[2] I. Dotlić and R. Kohno. Performance analysis of Impulse Radio Ultra-Wideband differential detection schemes for Body Area Networks. In IEEE 21st International Symposium on Personal, Indoor and Mobile Radio Communications Workshops (PIMRC Workshops), pages $72-77$, September 2010.

[3] I. Dotlić and R. Kohno. Application of IEEE 802.15.6 IR-UWB physical layer for medical BAN. International Journal of Ultra Wideband Communications and Systems, 2(2):104-115, September 2011.

[4] I. Dotlić and R. Kohno. Preamble Structure and Synchronization for IEEE 802.15.6 Impulse-Radio Ultra-Wideband Physical Layer. In 5th International Symposium on Medical Information and Communication Technology, ISMICT, March 2011.

[5] I. Dotlic and R. Miura. Sample-wise differential detection of impulse-radio ultra-wideband pulse position modulated symbols. In 6th International Symposium on Medical Information and Communication Technology (ISMICT), pages 1-4, 2012.

${ }^{1}$ see $[2-5]$. 


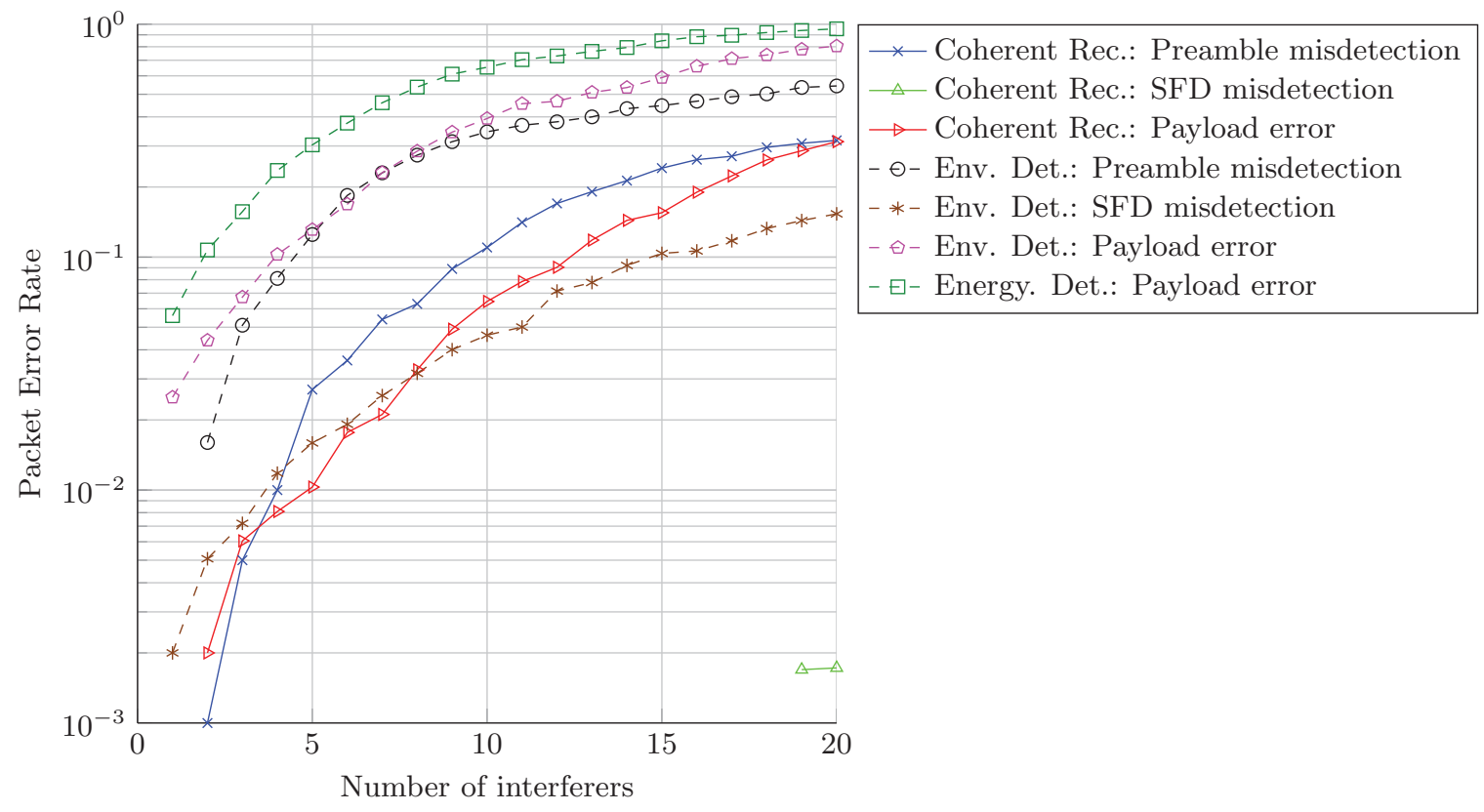

Figure 8: Performance of receiver architectures considered in Multiple Access Interference at data rate of $218 \mathrm{Kbps}$.

[6] P. W. Kinney, V. Brethour, J. Bain, P. Houghton,

J. Lampe, V. Brethour, Z. Sahinoglu, P. Orlik,

I. Lakkis, R. Hach, K.-K. Lee, M. Welborn, J. Bain,

B. A. Rolfe, M. Welborn, C. Gentile, and

M. McLaughlin. IEEE Standard for PART 15.4:

Wireless MAC and PHY Specifications for Low-Rate Wireless Personal Area Networks (LR-WPANs):

Amendment 1: Add Alternate PHY.

[7] D. Lewis. IEEE P802.15.6-2012 Standard for Body Area Network. IEEE 802.15 WPAN, February 2012.

[8] H.-B. Li, J. Schwoerer, Y. Yoon, J. F. W. Yang, K. Sayrafian, D. Miniutti, and D. Lewis. IEEE 802.15.6 Regulation Subcommittee Report. IEEE 802.15 WPAN, (IEEE 802.15-08-0034-15-0006), May 2010.

[9] M. McInnis. IEEE Standard for Local and Metropolitan area networks Part 15.4: Low-Rate Wireless Personal Area Networks (WPANs), Amendment: Active Radio Frequency Identification. IEEE 802.15 WPAN, April 2012.

[10] J. G. Proakis. Digital Communications. McGraw-Hill, New York, 4th edition, 2001.

[11] K. Y. Yazdandoost. Channel Model for Body Area Network (BAN). IEEE 802.15 WPAN, (IEEE P802.15-08-0780-12-0006), November 2010. 


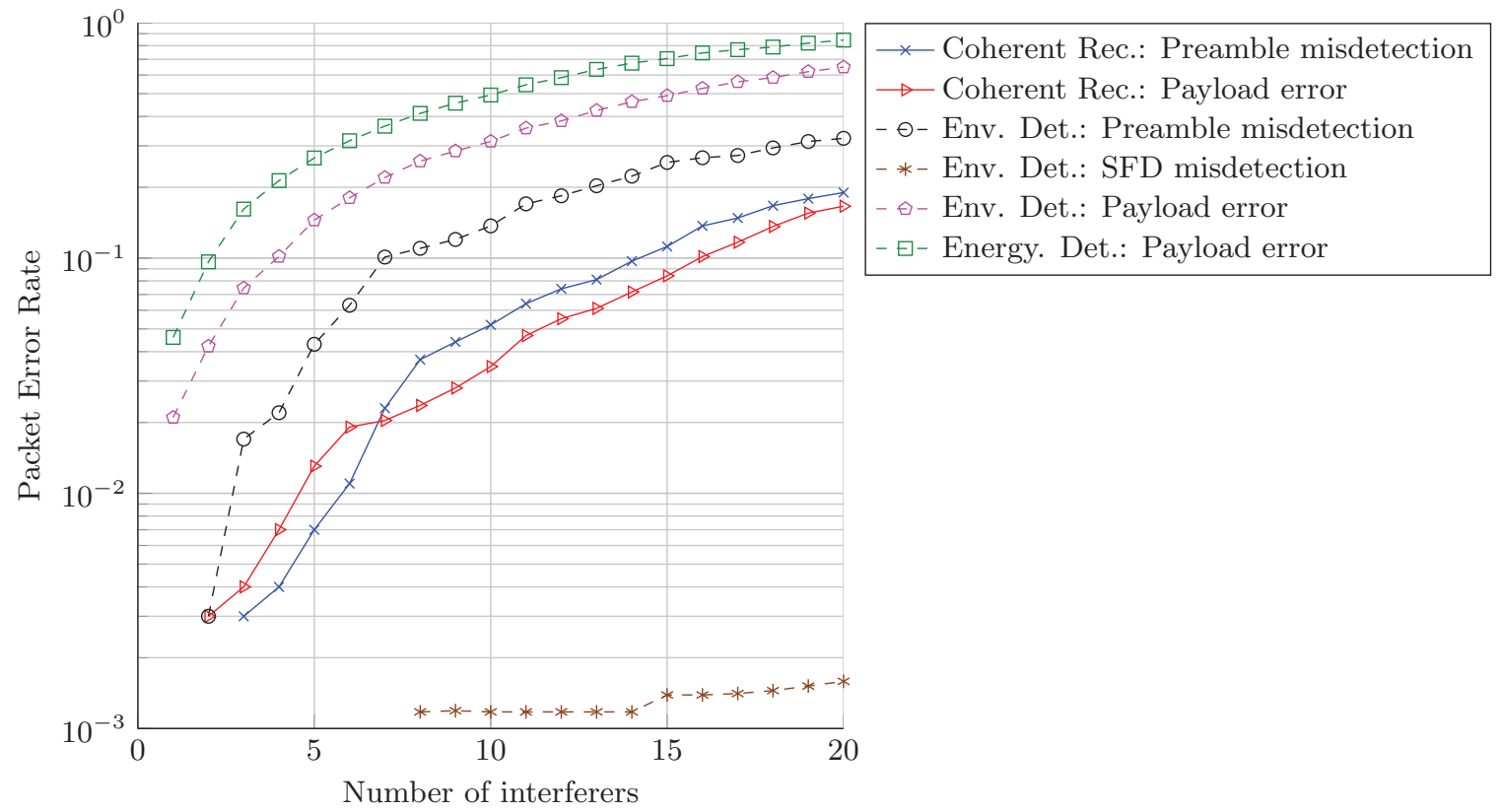

Figure 9: Performance of receiver architectures considered in Multiple Access Interference at data rate of $436 \mathrm{Kbps}$. 